\title{
Carbon isotopic abundance ratios in S-type stars *
}

\author{
G. Wallerstein ${ }^{1}$, B. Balick ${ }^{1}$, J. Alcolea ${ }^{2}$, V. Bujarrabal ${ }^{2}$, and A. D. Vanture ${ }^{3}$ \\ 1 Astronomy Department, University of Washington, Seattle WA 98195-1580, USA \\ e-mail: [walleg; balick]@uw.edu \\ 2 Observatorio Astronómico Nacional, Apartado 1143, Alcalá de Henares, 28800 Madrid, Spain \\ e-mail: [alcolea; v.bujarrabal]@oan.es \\ ${ }^{3}$ Physical Sciences Dept., Everett Community College, Everett, WA, USA \\ e-mail: avanture@everettcc .edu
}

Received 24 June 2010 / Accepted 26 September 2011

\begin{abstract}
We have used the IRAM $30 \mathrm{~m}$ telescope on Pico de Veleta to observe 11 S-type stars in the $J=1-0$ and $J=2-1$ transitions of both ${ }^{12} \mathrm{C}^{16} \mathrm{O}$ and ${ }^{13} \mathrm{C}^{16} \mathrm{O}$ in order to derive the ${ }^{12} \mathrm{CO} /{ }^{13} \mathrm{CO}$ ratio in their circumstellar envelopes. Detections of high signal to noise were achieved for 7 stars. A mean isotope ratio of $35.3 \pm 6.2$ was found. This ratio fits between the ratio for late $\mathrm{M}$ stars of about $12 \pm 3$ and that of carbon stars of $58 \pm 17$. Hence, we confirm the often proposed evolutionary sequence from late $\mathrm{M}$ through type $\mathrm{S}$ to carbon stars. Mass loss rates of $5 \times 10^{-8}$ to $3 \times 10^{-6} M_{\odot} \mathrm{y}^{-1}$ are derived from the present CO data using a simple uniform outflow model with line-dependent opacities, in very good agreement with previous mass-loss estimates.
\end{abstract}

Key words. stars: AGB and post-AGB - stars: abundances

\section{Introduction}

Stars of type $\mathrm{S}$ were first recognized as a separate spectral class due to the presence of $\mathrm{ZrO}$ bands in addition to those of $\mathrm{TiO}$ in the spectra of cool giants (Merrill 1922). Merrill and others found that lines of many other heavy elements of the 4th and 5th rows of the periodic table are conspicuously enhanced in S stars. Merrill's (1952) discovery of the unstable element technetium in $\mathrm{S}$ stars and its explanation by Cameron (1955) as a result of neutron captures by lighter species opened the way to the introduction of the s-process as defined and discussed by Burbidge et al. (1957).

For a full background on the evolution of asymptotic giant branch (AGB) stars see Worley et al. (2010, especially the contribution by A. Chieffi). As stars of about $1-8 M_{\odot}$ evolve beyond the first red giant branch their central regions convert ${ }^{4} \mathrm{He}$ to ${ }^{12} \mathrm{C}$ via the triple-alpha reaction. For sufficiently small masses, less than about $1.5 M_{\odot}$, He burning starts with a "flash" due to degeneracy and continues to convert helium to carbon quiescently. More massive stars, up to about $8 M_{\odot}$, burn He steadily in their cores. As the core becomes almost completely ${ }^{12} \mathrm{C}$ (with some ${ }^{16} \mathrm{O}$ in the most massive stars) the core shrinks and heats to the point at which the boundary between the ${ }^{12} \mathrm{C}$ and the ${ }^{4} \mathrm{He}$ can become active and shell burning can convert ${ }^{4} \mathrm{He}$ to ${ }^{12} \mathrm{C}$. Due to the high temperature sensitivity of the 3 -alpha reaction the rate rises very rapidly in what is called a "shell flash". The large temperature gradient induces rapid convection which mixes the fresh ${ }^{12} \mathrm{C}$ into higher layers. As the ${ }^{12} \mathrm{C}$ is convected to the $\mathrm{He}-\mathrm{H}$ boundary some of the ${ }^{12} \mathrm{C}$ is converted to ${ }^{13} \mathrm{C}$ by proton capture. This is the source of the ${ }^{13} \mathrm{C}$ that is responsible for the free neutrons via the ${ }^{13} \mathrm{C}(\alpha, \mathrm{n}){ }^{16} \mathrm{O}$ reaction. A wide range of elements including

* Based on observations carried out with the IRAM 30 m radiotelescope. IRAM is supported by INSU/CNRS (France), MPG (Germany) and IGN (Spain).
$\mathrm{Zr}$ are produced by successive neutron captures by Fe-peak elements. When further mixing brings these elements to the surface the atmospheric abundance of $\mathrm{Zr}$ is substantially enhanced and the star is called an S star.

During the mixing process, ${ }^{12} \mathrm{C}$ is enhanced in the stellar atmosphere in which the ratio of ${ }^{12} \mathrm{CO} /{ }^{13} \mathrm{CO}$ had been depressed during previous $\mathrm{CNO}$ cycling. As atmospheric ${ }^{12} \mathrm{C}$ is further enhanced in the upwelling of recently produced ${ }^{12} \mathrm{C}$ the total carbon abundance equals, and then exceeds, the oxygen abundance and the atmosphere contains carbon molecules rather than oxides. That defines a carbon star. In a large survey of carbon stars, Lambert et al. (1986) found a range in ${ }^{12} \mathrm{C} /{ }^{13} \mathrm{C}$ from 20 to 90 with the exception of a few stars (known as $\mathrm{J}$ stars) with ratios near 3-4. Optical spectra of M giants by Luck \& Lambert (1982) and Lambert \& Smith (1990) have found ${ }^{12} \mathrm{C} /{ }^{13} \mathrm{C}$ ratios in the range of 7 to 23 with a mean of 15 . This shows a small excess of ${ }^{13} \mathrm{C}$ above the expected and observed ratio of 20 after the first dredge-up as a star evolves up the red giant branch for the first time. Similarly, in a survey of 18 additional $\mathrm{M}$ giants, mostly of types M4-M6, Brown and Wallerstein (unpublished) found a range of the ${ }^{12} \mathrm{C} /{ }^{13} \mathrm{C}$ isotope ratio of $8-18$ with a mean of $12 \pm 3$.

This shows that most of the carbon that has been added to the atmosphere during stellar evolutionary phases that precede the formation of carbon stars, has been ${ }^{12} \mathrm{C}$. Yet available observations provide only sparse coverage in the gap from $\mathrm{M}$ giants to carbon stars. In this paper we endeavor to fill this gap with ${ }^{12} \mathrm{C} /{ }^{13} \mathrm{C}$ values measured in the molecular gas in the ejected envelopes of $\mathrm{S}$ stars.

It is difficult to derive the ${ }^{12} \mathrm{C} /{ }^{13} \mathrm{C}$ ratios from optical spectra of S stars, though some stars have been analyzed using the $\mathrm{CN}$ lines near $8000 \AA$ and the CO lines in the near IR. In early M giants, there are $\mathrm{CN}$ bands near $8000 \AA$ which show significant isotopic shifts, and carbon stars show both $\mathrm{C}_{2}$ and $\mathrm{CN}$ bands. For the cooler $\mathrm{S}$ stars, with which we are concerned here, the 
Table 1. Target stars.

\begin{tabular}{lccccccc}
\hline \hline Star & $\begin{array}{c}\text { Spectral } \\
\text { type }\end{array}$ & Reference & Li & Tc & $\begin{array}{c}\text { Puls. period } \\
\text { (days) }\end{array}$ & $M_{K}$ & $\begin{array}{c}\text { Distance } \\
(\mathrm{pc})\end{array}$ \\
\hline W Aql $^{a}$ & S6,8 & 3 & $\ldots$ & $\ldots$ & 490 & -8.33 & 360 \\
R And $^{b}$ & S5-7,5 & 3 & No & Yes & 409 & -8.06 & 440 \\
TV Aur & S5,6 & 3 & Yes & Yes & 182 & -5.65 & 330 \\
AA Cam & M5 S & 4 & No & Yes & Irr & -5.65 & 250 \\
S Cas $^{c}$ & S5,4 & 1 & Yes & $\ldots$ & 612 & -8.67 & 910 \\
T Cet & M5-6S & 4 & No & Yes & 159 & -5.65 & 90 \\
R Cyg $^{d}$ & S6,6 & 3 & Yes & Yes & 426 & -8.12 & 550 \\
$\chi_{\text {Cyg }}{ }^{e}$ & S7,1 & 2 & No & Yes & 408 & -8.06 & 180 \\
ST Her & M6.5S & 3 & No & Yes & 148 & -5.65 & 300 \\
Y Lyn $^{f}$ & M5S & 4 & No & Yes & 110 & -5.65 & 100 \\
\hline & S5.5,6 & 3 & Yes & Yes & 395 & -8.00 & 790 \\
\hline
\end{tabular}

Notes. ${ }^{(a)}$ Also classified as S7, 1 (Udry et al. 1998). ${ }^{(b)}$ Tc first seen in this star (Merrill 1952). ${ }^{(c)}$ Star too faint in the blue to observe for Tc. One of the longest period Mira stars, very strong Li line. ${ }^{(d)}$ Called "pure S" because TiO is absent. Now alternating between high and low maxima. ${ }^{(e)}$ Atmosphere shows multiple layers (Wallerstein 1985). ${ }^{(f)}$ Very strong Li line.

References. (1) Ake (1979); (2) Keenan (1954); (3) Keenan \& Boeshaar (1980); (4) Stephenson (1976).

$\mathrm{TiO}, \mathrm{ZrO}$ and $\mathrm{LaO}$ bands blend with the $\mathrm{CN}$ bands to make them essentially unusable.

It is necessary to use near IR or millimeter spectra where circumstellar CO transitions are observable in stars with envelopes formed by the stellar wind. The CO lines in the $\mathrm{mm}$ region are formed far out in the stellar envelope rather than in the photosphere where the optical and near-IR lines are formed. With modern technology, radio observations of ${ }^{12} \mathrm{CO}$ and ${ }^{13} \mathrm{CO}$ emission lines are becoming routine. Lines from ${ }^{13} \mathrm{CO}$ are weaker than those from ${ }^{12} \mathrm{CO}$ but generally detectable nonetheless. The ${ }^{12} \mathrm{CO}$ lines are bright, though saturation can be an issue. In some cases, this can be avoided by using higher rotational transitions. Alternatively, radiative transfer in spherical winds can be used. We have pursued the latter course.

In this study, we have observed the $\mathrm{CO}$ isotopes with the IRAM 30-m telescope in the $J=1-0$ and $J=2-1$ transitions of $11 \mathrm{~S}$ stars as well as the calibration star CW Leo.

\section{Target selection and observations}

Targets were selected from an investigation of $\mathrm{Li}$ and $\mathrm{Tc}$ in Galactic S stars by Vanture et al. (2007), modified by the necessity that they be observable when the telescope was assigned to us. All of the stars show the Tc lines, demonstrating that they are intrinsic $S$ stars rather than extrinsic $S$ stars that have received heavy element-enhanced material from a now defunct companion. Possible exceptions are S Cas and W Aql, which we found to be too faint in the $4200 \AA$ region in our search for Tc. $\mathrm{S}$ Cas has the longest period and one of the strongest Li lines among the S stars. Lambert et al. (1995) state that it is most likely an intrinsic S Star. With its period of 490 days and spectral type of S3, 9-S6, 9, W Aql is similar to S Cas. In Table 1 we show the most important properties of the observed stars. Useable trigonometric parallaxes are available for only 3 stars observed by Hipparcos (Van Leeuwen 2008). For all stars we list the derived $M_{K}$ magnitude using the period luminosity relation for Mira-type stars (Whitelock et al. 2008, Fig. 1), which has the form

$M_{K}=\rho[\log P-2.38]+\delta$,

where $M_{K}$ is the absolute $K$ magnitude, $P$ the pulsation period, and $\delta$ the zero-point of the $M_{K}-P$ relation, which has slope $\rho$.
For non-Mira - i.e. semi-regular - variables we use the value suggested by Guandalini \& Busso (2008), viz -5.64 for $M_{K}$. To estimate the distance, we accept the $K$ magnitude as listed in SIMBAD, since the pulsation amplitude in $K$ is not large. A comparison of the period-luminosity method with the HIPPARCOS parallaxes for 3 stars indicates that an uncertainty of about a factor of 2 is likely. Possible errors in distance have only a small impact on the derivation of the carbon isotope ratios.

The observations have been carried out with the IRAM $30 \mathrm{~m}$ millimeter radio-telescope (30 m-MRT), at an altitude of $2850 \mathrm{~m}$ located on Pico de Veleta (southern Spain). We have observed the two lowest rotational transitions, $J=2-1$ and $J=1-0$, of both ${ }^{12} \mathrm{C}^{16} \mathrm{O}$ and ${ }^{13} \mathrm{C}{ }^{16} \mathrm{O}$ isotopic substitutions of carbon monoxide $\left({ }^{12} \mathrm{CO}\right.$ and ${ }^{13} \mathrm{CO}$ hereafter $)$, at wavelengths of 2.6 and $1.3 \mathrm{~mm}$. We have assumed rest frequencies of 110.201353 and $115.271202 \mathrm{GHz}$ for the $J=1-0$ line of ${ }^{13} \mathrm{CO}$ and ${ }^{12} \mathrm{CO}$, and of 220.398681 and $230.537990 \mathrm{GHz}$ for the $J=2-1$ line of ${ }^{13} \mathrm{CO}$ and ${ }^{12} \mathrm{CO}$, respectively.

The observations were performed in three runs, in June and November 2007, and in January 2009. During the first two runs, the weather conditions were mediocre to poor: precipitable water vapor content (PWV) from 1.0 to $10 \mathrm{~mm}$, and total system noise equivalent temperatures ( $\left.T_{\text {sys }}\right)$ of $100-300 \mathrm{~K}$ and $200-700 \mathrm{~K}$ at 2.6 and $1.3 \mathrm{~mm}$ respectively. On the contrary, weather was excellent on the last observing run, with PWV from $<0.5$ to $2 \mathrm{~mm}$ and $T_{\text {sys }}$ of $150-200$ and $250-400 \mathrm{~K}$ at 2.6 and $1.3 \mathrm{~mm}$ respectively. The data presented here has been calibrated in units of (Rayleigh-Jeans-equivalent) main beam temperature (corrected for the atmospheric attenuation), $T_{\mathrm{mb}}$, using the standard chopper wheel method. The temperature scale is set by observing hot and cold loads at ambient and liquid nitrogen temperatures. Correction for the antenna coupling to the sky and other losses have been applied using the latest values for these parameters measured at the telescope.

Finally, we accounted for the sky attenuation from the values of a weather station, the measurement of the sky emissivity and a numerical model for the atmosphere at Pico de Veleta. Calibration scans (observation of the hot and cold loads, and of the blank sky) were performed typically every $20 \mathrm{~min}$. For the conversion into $T_{\mathrm{mb}}$ units, we have adopted values of the forward and main beam efficiencies of the telescope of 0.95 and 0.70 , respectively, for the $J=1-0$ lines, and of 0.91 and 0.58 for the $J=2-1$ lines. 

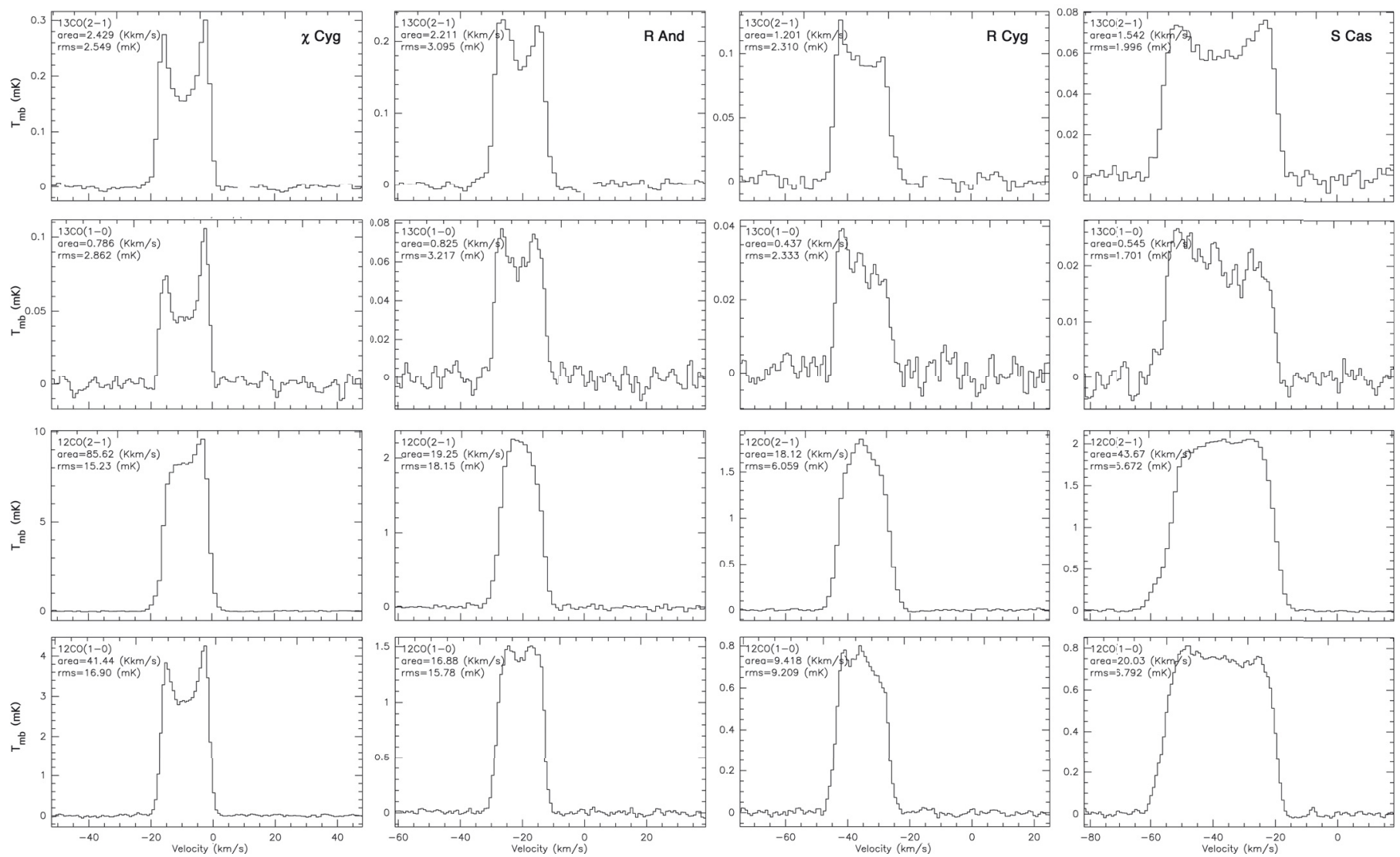

Fig. 1. Montage of $\mathrm{CO}$ spectra of $\mathrm{S}$ stars in which ${ }^{13} \mathrm{CO}$ lines are visible. The main beam (observed) temperature, $T_{\mathrm{mb}}$, is the ordinate in units of $\mathrm{mK} .{ }^{13} \mathrm{CO}$ and ${ }^{12} \mathrm{CO}$ transitions are identified in the upper left corners of each panel along with the integrated line fluxes and the rms of $T_{\mathrm{mb}}$. More saturated lines tend to exhibit flatter profile tops. Note that the relatively unsaturated ${ }^{13} \mathrm{CO}$ lines invariably show double peaks. This is characteristic of uniform spherical expansion in a partially resolved envelope (see text).

In addition to the method just described, during the runs we interspersed observations of strong (i.e. "template") CO emitters to test the consistency of the calibration procedure. We believe that the final relative calibration between the different sources in our program is better than $10 \%$, while the errors in the absolute calibration should be smaller than about $20 \%$, after correcting for the variations observed in the strength of these $\mathrm{CO}$ template emitters.

The pointing of the telescope was checked, and corrected if necessary, when changing the observed source or every two hours, by cross-scan observations of strong continuum sources. From the results of the pointing scans we estimate that the overall pointing error during the observations is less than $2^{\prime \prime}$, which has negligible influence in the calibration even at the highest frequency observed. The observations were done by wobbling the secondary mirror by $2^{\prime}$ at a $0.5 \mathrm{~Hz}$ rate. This method provides very stable and flat spectral baselines, so after data averaging linear baselines have been fitted and subtracted from the final spectra.

At the telescope, we used the old A and B dual (3 and $1.3 \mathrm{~mm}$ ) band receivers to observe simultaneously all four $\mathrm{CO}$ lines. In the last run, since we only observed ${ }^{13} \mathrm{CO}$, we recorded the two lines in two receivers at the same time, improving the $S / N$ ratio by $\sqrt{2}$ after averaging. The receivers worked in SSB mode, with typical noise temperatures of 80 and $150 \mathrm{~K}$ at 2.6 and $1.3 \mathrm{~mm}$ respectively. As spectral back-end we used a $1 \mathrm{MHz}$ resolution filter-bank for the $1.3 \mathrm{~mm}$ lines, and the VESPA auto-correlator in its $312.5 \mathrm{kHz}$ resolution configuration for the $J=1-0$ lines at $2.6 \mathrm{~mm}$. This resulted in original spectral resolutions of 1.3 and $0.85 \mathrm{~km} \mathrm{~s}^{-1}$ for the $J=2-1$ and $J=1-0$ lines respectively.

In all cases, we observed just a single pointing towards the star position. The mean half-power beam width (HPBW) at the $30 \mathrm{~m}$-MRT at 2.6 and $1.3 \mathrm{~mm}$ are $23^{\prime \prime}$ and $11.5^{\prime \prime}$ respectively. Depending on the relative extent of the $\mathrm{CO}$ envelope with respect to the main beam size of the telescope, a correction for the effect of the convolution of the molecular emission with the telescope beam has to be introduced. Corrections due to this effect mostly affect large envelopes in optically thick lines $\left(J=2-1{ }^{12} \mathrm{CO}\right)$ and can be estimated from the shape of all $4 \mathrm{CO}$ lines.

\section{Results}

Opacity effects in the radiation transfer of the ${ }^{12} \mathrm{CO}$ and, to a smaller degree, the ${ }^{13} \mathrm{CO}$ lines force the use of a model to interpret the observational data. The double-horned profile shapes that characterize the ${ }^{13} \mathrm{CO}$ and the likelihood of steady mass loss in $\mathrm{S}$ stars motivate the use of a uniformly expanding spherical model for estimating mass loss rates and ${ }^{12} \mathrm{CO} /{ }^{13} \mathrm{CO}$ abundance ratios from our observations. Fortunately, as we will see, our method is simple and robust thanks to the favorable properties of the excitation and emission of the $\mathrm{CO}$ low- $J$ transitions.

We follow the theoretical intensity predictions by Knapp \& Morris (1985). These authors performed grids of calculations that give values of the ${ }^{12} \mathrm{CO} J=1-0$ intensity for values of the mass-loss rate, $\dot{M}$, the distance $D$ to the star, the CO abundance $X(\mathrm{CO})$ relative to $\mathrm{H}_{2}$ (assumed to be $6 \times 10^{-4}$ ), the circumstellar 
expansion velocity, $V_{\text {exp }}$, and the outer radius of the CO-rich envelope, $R_{\mathrm{CO}}$, beyond which the $\mathrm{CO}$ dissociates.

In general, the excitation and emission of low- $J \mathrm{CO}$ lines is very easy to describe physically, due to the simple level structure of this molecule and that the populations of all of the low-lying levels are thermalized even for relatively low densities. For example, the $J=1-0$ transition requires an excitation temperature of only $5.5 \mathrm{~K}$, to be excited. Thus, for simplicity, we will base our estimates on observations of this line.

Another advantage of using $J=1-0$ profiles is that the extended $\mathrm{CO}$ envelope is rarely larger than our telescope beam, $\sim 23^{\prime \prime}$ at $115 \mathrm{GHz}$. As we will explain below, we have applied corrections to the line intensities for the few sources that may be partially resolved, in order to introduce the resulting intensities in the estimates of the mass-loss rates from the theoretical grids. In any case, those corrections are almost always very modest and their uncertainty has little effect on our results.

In most cases, the parabolic shapes and in general central peaks in the ${ }^{12} \mathrm{CO}$ profiles are a clear sign of a significant degree of opacity in the expanding envelope (see general discussion by Olofsson et al. 1982), which must be taken into account in the calcultions. Since it is possible that ${ }^{12} \mathrm{CO} /{ }^{13} \mathrm{CO}<10$, opacity effects for ${ }^{13} \mathrm{CO}$ profiles cannot be dismissed. However, for those profiles with two horns or oddly shaped profile cores - as in most of our ${ }^{13} \mathrm{CO}$ profiles - we can assume optically thin lines. These general trends have been widely confirmed by observations and theoretical detailed models (see e.g. Ramstedt et al. 2008; Teyssier et al. 2006).

In Fig. 1 we present examples of our ${ }^{12} \mathrm{CO}$ and ${ }^{13} \mathrm{CO}$ profile shapes. Four stars in our survey show signs of optically thick ${ }^{12} \mathrm{CO}$ emission: W Aql, R And, S Cas, and R Cyg. Of these, high opacity is confirmed in W Aql and S Cas (Ramstedt et al. 2009; Nyman et al. 1992). So we will apply the full optically thick case for them. For R And and R Cyg, in which the opaque nature of the lines is less clear, we will adopt values averaged between those obtained from the optically thin and thick cases. As discussed in Appendix A, the estimated ${ }^{12} \mathrm{CO} /{ }^{13} \mathrm{CO}$ abundance ratios are in fact not very different in both cases.

For optically thick lines, the comparison of the observed line profiles with the models we use to estimate mass-loss rates requires an estimate of the profile's central temperature of the $J=1-0$ lines for a beam that includes the entire CO outflow zone. For relatively small sources, these values are directly given by our observations. In some cases, in which the shell is partially resolved, some correction must be applied. Such corrections are estimated assuming that all the emission at velocities well separated from the systemic one is well picked up by our single-point observations, since at these velocities the line emission comes from an angularly compact region along the central line of sight that can be assumed to be always unresolved. The peak intensity was then estimated by applying to our observations the relative increase between the central peak and the adjacent velocities found in the profiles of our targets obtained by Ramstedt et al. (2009) and Nyman et al. (1992), who used a wider beam. Even so, the procedure is a bit risky for $\mathrm{W}$ Aql and S Cas owing to possible uncertainties in our respective intensity calibrations.

This problem is less important in optically thin cases, in which the line peak always appears at extreme velocities, leading in the limit to clear two-horn profiles. We recall that the brightness distribution at those extreme velocities, which comes from an angularly compact region along the central line of sight, can be assumed to be unresolved. Therefore, our peak intensities should accurately give the intensity measure needed to be included in the theoretical estimates.
We note that the expected outer CO radii adopted by Knapp $\&$ Morris (1985), as due to CO photodissocition, are now understood to be too large owing to new better studies of dissociation by the interstellar UV radiation field. Detailed calculations of the CO photodissociation radius were performed by Mamon et al. (1988) for the relevant cases, taking into account the shielding by dust and by the $\mathrm{CO}$ molecule itself. The resulting predicted radii were parameterized as a function of the mass loss rate, the expansion velocity, and the relative abundance by Planesas et al. (1990), see also Loup et al. (1993). Accordingly, we introduced the photodissociation radius calculated in this way in the grids by Knapp \& Morris, in our calculation of mass-loss rates and ${ }^{12} \mathrm{CO} /{ }^{13} \mathrm{CO}$ abundances. Since the radius also depends on $\dot{\mathrm{M}}$ and distance, the procedure needs to be applied iteratively.

We note that the outer ${ }^{13} \mathrm{CO}$ radius is practically identical to that of ${ }^{12} \mathrm{CO}$ (Mamon et al. 1988) because, although ${ }^{13} \mathrm{CO}$ is more easily photodissociated by the interstellar UV field, subsequent fractionation reactions tend to reform ${ }^{13} \mathrm{CO}$. To first order, the abundance is kept practically constant up to the photodissociation radius of ${ }^{12} \mathrm{CO}$. From this radius, both molecules are dissociated and their abundances drop very fast.

${ }^{13} \mathrm{CO} J=1-0$ is always found to be optically thin in our targets, as expected for the typical low abundance of this species in circumstellar envelopes around AGB stars. However, we note that opacity corrections could also be applicable to ${ }^{13} \mathrm{CO}$ emission in a few extreme cases of small ${ }^{12} \mathrm{CO} /{ }^{13} \mathrm{CO}$ values, since the excitation of both isotopic substitutions is very similar. In the unrealistic case where ${ }^{12} \mathrm{CO} \approx{ }^{13} \mathrm{CO}$ the predicted intensities of their $J=1-0$ lines are the same aside from a small difference $\approx(115 \mathrm{GHz} / 110 \mathrm{GHz})^{2}, \sim 9 \%$. In the more general case where ${ }^{12} \mathrm{CO} /{ }^{13} \mathrm{CO}>10$, this type of uncertainty is significantly smaller than the general uncertainty in the theoretical analysis of $\mathrm{CO}$ data. Thus, we have not tried to introduce any correction when applying the theoretical grids to ${ }^{13} \mathrm{CO}$.

Finally, we note that the value of $X\left({ }^{12} \mathrm{CO}\right)$ has been very well studied in circumstellar envelopes. But even if $X\left({ }^{12} \mathrm{CO}\right)$ is somehow unique for $\mathrm{S}$ stars, the adopted value is not critical in our estimates of the ${ }^{12} \mathrm{CO} /{ }^{13} \mathrm{CO}$ abundance, because any change in $X\left({ }^{13} \mathrm{CO}\right)$ affects the determination of $\dot{M}$ yielding a change in $X\left({ }^{12} \mathrm{CO}\right)$ in the same sense.

In summary, using the above procedure, we have first estimated the mass-loss rate and the $\mathrm{CO}$ photodissociation radius from our ${ }^{12} \mathrm{CO} J=1-0$ profiles. We then used these results to estimate the abundance of ${ }^{13} \mathrm{CO}$ and therefore the ${ }^{12} \mathrm{CO} /{ }^{13} \mathrm{CO}$ relative abundance.

Typical CO spectra for $\chi$ Cyg, R And, R Cyg, and S Cas are shown in Fig. 1. For T Sgr and the short period stars T Cet, AA Cam, and TV Aur, the $S / N$ was insufficient to derive useful isotope ratios. The peak line intensities as well as the mass loss rates and $\mathrm{CO}$ isotopic ratios derived from these spectra are shown in Table 2. Obviously the ${ }^{13} \mathrm{CO}$ lines are partially saturated in some of the target stars. The final column of Table 2 shows how we treated these lines in the model analysis. In two cases, it is not clear if the lines are predominantly optically thick or thin, so we performed two calculations and adopted the average values. The mass-loss estimates are from our data and our simple modeling (based on the calculation grids by Knapp \& Morris 1985), and derived ${ }^{12} \mathrm{CO} /{ }^{13} \mathrm{CO}$ abundance ratios.

Based on the 5 Miras and 2 semi-regular variables for which measurements are feasible, the mean isotopic ratio of ${ }^{12} \mathrm{C} /{ }^{13} \mathrm{C}$ is 36.1 after correction for opacity effects. The dispersion in ${ }^{12} \mathrm{C} /{ }^{13} \mathrm{C}$ is 7.5 with extreme values between $\sim 25$ and $\sim 46$ for objects in which both lines were detected. The mean isotopic abundance for our sample is close to the geometric mean for $\mathrm{M}$ 
Table 2. Peak line intensities and other parameters derived from our observations.

\begin{tabular}{lccccc}
\hline \hline Star & $\begin{array}{c}T_{\mathrm{mb}}\left({ }^{12} \mathrm{CO}\right) \\
(\mathrm{K})\end{array}$ & $\begin{array}{c}T_{\mathrm{mb}}\left({ }^{13} \mathrm{CO}\right) \\
(\mathrm{K})\end{array}$ & $\begin{array}{c}\dot{M} \\
\left(M_{\odot} \mathrm{y}^{-1}\right)\end{array}$ & ${ }^{12} \mathrm{CO} /{ }^{13} \mathrm{CO}$ & Model \\
\hline W Aql & $4.5^{*}$ & 0.204 & $2.010^{-5}$ & 30.9 & op. thick \\
R And & $1.6^{*}$ & 0.075 & $1.210^{-6}$ & 43.0 & op. thick \\
R And & 1.5 & 0.075 & $7.210^{-7}$ & 20.0 & $\begin{array}{l}\text { op. thin } \\
\text { R And }\end{array}$ \\
TV Aur & 0.067 & $<0.028$ & $7.210^{-8}$ & 33.3 & average \\
AA Cam & 0.084 & $<0.016$ & $2.910^{-8}$ & $>5.2$ & op. thin \\
S Cas & $0.87^{*}$ & 0.019 & $6.910^{-6}$ & 46.2 & op. thin \\
T Cet & 0.22 & $<0.064$ & $8.210^{-8}$ & $>3.4$ & op. thick \\
R Cyg & $0.85^{*}$ & 0.039 & $1.210^{-6}$ & 43.6 & op. thick \\
R Cyg & 0.8 & 0.039 & $7.310^{-7}$ & 20.5 & op. thin \\
R Cyg & & & & 33.9 & average \\
$\chi$ Cyg & 4.25 & 0.11 & $4.710^{-7}$ & 38.6 & op. thin \\
ST Her & 0.43 & 0.017 & $2.010^{-7}$ & 25.3 & op. thin \\
Y Lyn & 0.78 & 0.02 & $2.810^{-7}$ & 39.0 & op. thin \\
T Sgr & 0.13 & $<0.034$ & $2.810^{-7}$ & $>3.8$ & op. thin \\
\hline average $\pm \sigma$ & & & $35.3 \pm 6.2$ & \\
\hline
\end{tabular}

Table 3. Velocity measurements (ordered by pulsation period).

\begin{tabular}{lcccc}
\hline \hline Star & $\Delta V_{\text {FWHM }}$ & $V_{\mathrm{CO}}$ & $V_{\mathrm{Opt}}$ & Reference \\
\hline S Cas & 37 & -38 & -32 & GCRV (1953) \\
W Aql & 32 & -13 & -18 & GCRV (1953) \\
R Cyg & 18 & -36 & -25 & GCRV (1953) \\
R And & 17 & -21 & -11 & GCRV (1953) \\
X Cyg & 20 & -10 & -2 & GCRV (1953) \\
T Sgr & 18 & -5 & +2 & GCRV (1953) \\
TV Aur & 10 & +20 & $\ldots a$ & $\ldots$ \\
T Cet & 13 & +30 & +29 & GCRV (1953) \\
ST Her & 18 & -23 & -21 & Famaey et al. (2005) \\
Y Lyn & 18 & +3 & +7 & Feast et al. (1972) \\
AA Cam & 8 & -50 & -48 & Famaey et al. (2005) \\
\hline
\end{tabular}

Notes. All velocities (in $\mathrm{km} \mathrm{s}^{-1}$ ) are heliocentric. ${ }^{(a)}$ No velocity data found in Simbad.

and carbon stars, suggesting the $\mathrm{S}$ stars fill the gap along the AGB between $\mathrm{M}$ and carbon stars.

In Table 3 we show the full width at half maximum (FWHM) of the strongest $\mathrm{CO}$ feature, the central velocity, the previously measured optical velocity, and the source for the latter. All velocities are heliocentric in $\mathrm{km} \mathrm{s}^{-1}$. Optical data are taken from SIMBAD, most of which come from the General Catalogue of Radial Velocities (Wilson 1953) and are usually based on the high-dispersion spectra obtained by P. W. Merrill with the Mt. Wilson spectrographs. These observations were based on spectra in the 4000-5000 $\AA$ region where the atmospheric opacity is high due to Rayleigh scattering by the $\mathrm{H}_{2}$ molecule. Thus, they represent the radial velocity at the upper layer of the atmosphere.

In Table 4 we show the estimated mass loss rates based on (1) sophisticated model fitting by Ramstedt et al. (2009) and (2) from our simple estimates. For our estimates we have adopted the same stellar parameters as Ramstedt et al. wherever possible. For some stars, we present calculations in both optically thin and thick cases. In some cases, when none of the values appear between brackets, it is not clear if the emission is optically thin or thick and the best estimate is an average of both. The asterisks indicate the stars in which we could not use 1-0 data from Ramstedt et al., and so the ratio between the estimates is affected by calibration uncertainties. The agreement of both methods is very satisfactory.
There is a noticeable correlation between the envelope expansion velocity and period that has long been known for Mira stars. We have also compared the $\mathrm{CO}$ and optical velocities. We find a systematic difference of $6.2 \pm 2.4 \mathrm{~km} \mathrm{~s}^{-1}$ for six regular Miras. In contrast, the difference is $1.75 \pm 1.0 \mathrm{~km} \mathrm{~s}^{-1}$ for the four semi-regulars. Both sets of differences are in the sense that velocities from spectra in the blue region are larger. Our difference of $6.2 \mathrm{~km} \mathrm{~s}^{-1}$ for the six Miras is almost the same as the $\mathrm{K}$ term of $5 \mathrm{~km} \mathrm{~s}^{-1}$ derived by Reid (1976).

The agreement is limited by uncertainties in the outflow speeds at the stellar surface where the optical lines arise and the cooler envelope outflow in which the molecular lines are formed and excited. That is, Mira atmospheres are dynamic in which rising and falling parcels of gas form radiative shocks during portions of the pulsation cycle (Hinkle et al. 1982).

\section{Discussion}

The $\mathrm{S}$ stars appear to be in an evolutionary stage on the asymptotic giant branch between the $\mathrm{M}$ giants and the carbon stars. During their evolution, ${ }^{12} \mathrm{C}$ produced in shell flashes is convected to the surface and the abundances of various heavy elements are enhanced by neutron captures. The neutrons are released by the reaction ${ }^{13} \mathrm{C}(\alpha, \mathrm{n}){ }^{16} \mathrm{O}$ which serves also to deplete the ${ }^{13} \mathrm{C}$ (Herwig, 2005; Palmerini et al. 2009). In late $\mathrm{M}$ giants, the ${ }^{12} \mathrm{C} /{ }^{13} \mathrm{C}$ ratio is about $12 \pm 3$ (Luck \& Lambert 1982; Smith \& Lambert 1990a,b; Wallerstein \& Morell 1994; Brown \& Wallerstein, unpublished). For carbon stars, excluding the $\mathrm{CH}$ stars and the J-type subspecies, the ${ }^{12} \mathrm{C} /{ }^{13} \mathrm{C}$ ratio ranges from 20 to 90 with a mean of $58 \pm 17$ (Lambert et al. 1986). Thus, the ${ }^{12} \mathrm{C} /{ }^{13} \mathrm{C}$ ratio of $\mathrm{S}$ stars fits nicely between that of late $\mathrm{M}$ giants and carbon stars, as is expected from the evolutionary sequence that has long been hypothesized.

In addition to the carbon isotopes, there are several features in the spectra of S stars that are sensitive to nuclear reactions in the stars themselves, as noted in Table 1. These are the presence and strength of the resonance line of $\mathrm{Li}$, the strength of the $\mathrm{Zr}$ lines and $\mathrm{ZrO}$ bands, and the presence of the Tc I lines. Each of these is affected by reactions in the stellar interior and mixing to the surface. Even if Tc is produced and mixed to the surface, its presence limits the time since its production to a few half-lives or less than a million years. 
Table 4. Comparison of mass loss estimates $\left(M_{\odot} \mathrm{yr}^{-1}\right)$.

\begin{tabular}{lccccc}
\hline \hline Star & Ramstedt et al. & $\begin{array}{c}\text { Simple estimate } \\
\text { (op. thick) }\end{array}$ & $\begin{array}{c}\text { Simple estimate } \\
\text { (op. thin) }\end{array}$ & Comments & Estimate ratio \\
\hline W Aql & $2.2 \times 10^{-6}$ & $2.6 \times 10^{-6}$ & & op. thick & 1.18 \\
R And & $6.6 \times 10^{-7}$ & $9.1 \times 10^{-7}$ & $5.6 \times 10^{-7}$ & op. thick? & 1.11 \\
AA Cam & $4 \times 10^{-8}$ & & $5.8 \times 10^{-8}$ & op. thin & $1.45^{*}$ \\
S Cas & $3.5 \times 10^{-6}$ & $3.2 \times 10^{-6}$ & $\left(2.2 \times 10^{-6}\right)$ & op. thick & 0.91 \\
T Cet & $4 \times 10^{-8}$ & & $4.9 \times 10^{-8}$ & op. thin & $1.23^{*}$ \\
R Cyg & $6.3 \times 10^{-7}$ & $8.0 \times 10^{-7}$ & $4.8 \times 10^{-7}$ & op. thick? & 1.02 \\
X Cyg & $3.8 \times 10^{-7}$ & $\left(4.8 \times 10^{-7}\right)$ & $2.7 \times 10^{-7}$ & op. thin & 0.71 \\
ST Her & $1.3 \times 10^{-7}$ & & $1.9 \times 10^{-7}$ & op. thin & $1.46^{*}$ \\
Y Lyn & $2.3 \times 10^{-7}$ & & $2.4 \times 10^{-7}$ & op. thin & 1.04 \\
T Sgr & $1.4 \times 10^{-7}$ & & $1.7 \times 10^{-7}$ & op. thin & 1.3 \\
\hline
\end{tabular}

${ }^{7} \mathrm{Li}$ is produced in red giants by the Cameron-Fowler (1971) process whereby ${ }^{4} \mathrm{He}+{ }^{3} \mathrm{He} \rightarrow{ }^{7} \mathrm{Be}$, which decays to ${ }^{7} \mathrm{Li}$ with a half-life of 53 days. It must be convected to lower temperatures before decaying or else the ${ }^{7} \mathrm{Li}$ is converted to two ${ }^{4} \mathrm{He}$ nuclei by proton capture. The ${ }^{12} \mathrm{C} /{ }^{13} \mathrm{C}$ ratio is strongly affected by proton capture by ${ }^{12} \mathrm{C}$ but the ${ }^{13} \mathrm{C}$ may capture another proton to make ${ }^{14} \mathrm{~N}$. At temperatures near $10^{8} \mathrm{~K}$, neutrons are released by ${ }^{13} \mathrm{C}(\alpha, \mathrm{n}){ }^{16} \mathrm{O}$ and are available to produce heavy elements such as the $\mathrm{Sr}, \mathrm{Y}, \mathrm{Zr}$ group as well as $\mathrm{Ba}$ and the lighter rare earths. This process is undoubtedly the source of the excess $\mathrm{Zr}$ and the Tc seen in S stars (Cameron 1955). In Table 1 we have shown the presence of each of these key species in our target stars.

Lithium appears in some galactic S stars (Vanture et al. 2007) as well as in S stars in the Magellanic Clouds (hereafter MC) (Smith \& Lambert 1989, 1990a,b; Plez et al. 1993). This discovery was a surprise as lithium is easily destroyed at the temperatures necessary to synthesize Tc and other post-iron peak elements via the s-process. The presence of $\mathrm{Li}$ in the $\mathrm{S}$ stars of the $\mathrm{MC}$ is nicely explained by the process of Hot Bottom Burning (HBB) (D' Antona \& Mazzitelli 1996; Mazzitelli et al. 1999; Sackmann \& Boothroyd 1992; Boothroyd et al. 1993). In HBB, ${ }^{7} \mathrm{Li}$ is produced in red giants by the Cameron-Fowler mechanism (Cameron \& Fowler 1971). The HBB theory makes specific predictions about the ${ }^{12} \mathrm{C} /{ }^{13} \mathrm{C}$ ratio in the photospheres of Li-rich AGB stars, namely the ratio should be low between 3 and 18 (Boothroyd et al. 1993).

There are three stars in our sample that exhibit both Tc and Li lines in their spectra, TV Aur, R Cyg and T Sgr (Vanture et al. 2007). A fourth star, S Cas, has a strong Li resonance line (Vanture et al. 2007; Lambert et al. 1995). Though no determination of the presence of Tc lines in the spectrum of S Cas exists in the literature, Lambert et al. (1995) argue that it is most likely an intrinsic $\mathrm{S}$ star based upon characteristics that it shares with other known intrinsic S stars. Therefore, it is expected that S Cas will exhibit Tc lines in its spectrum. The lithium line in S Cas is very strong (Lambert et al. 1995) and a comparison of its appearance as illustrated in Fig. 2 of Vanture et al. (2007) to that in the super-Li rich S star V441 Cyg as shown in Fig. 7 of Uttenthaler \& Lebzelter (2010) suggests that S Cas may also be a super-Li S star. The Li abundance of V441 Cyg $(\log \epsilon(\mathrm{Li})=4.4$; Uttenthaler $\&$ Lebzelter 2010) as well as that of T Sgr $(\log \epsilon(\mathrm{Li})=4.2$; Abia et al. 1991), on the abundance scale of $\log \epsilon(\mathrm{H})=12.0$, are in agreement with the predictions of HBB.

Of the four stars in our sample which can be classified as intrinsic $\mathrm{S}$ stars with enhanced $\mathrm{Li}$ abundances the lower limit of the ${ }^{12} \mathrm{C} /{ }^{13} \mathrm{C}$ ratio of TV Aur and T Sgr do not conflict with the predictions of HBB. However, the carbon isotope ratios in $\mathrm{S}$ Cas and R Cyg are well above those predicted by HBB theory. If $\mathrm{R}$ Cyg and S Cas have lithium abundances comparable to those of V441 Cyg and T Sgr (and the evidence that this is true for $\mathrm{S}$ Cas at least is compelling) then our results present a problem for HBB theory.

In addition, HBB theory predicts that these stars should have $M_{\text {bol }}<-6.0$ and the bolometric magnitudes predicted using Eq. (1) are well above this limit for the four stars with Li. However, it should be noted that predicting the $M_{\text {bol }}$ of Galactic $\mathrm{S}$ stars is not an exact science. For example, Uttenthaler \& Lebzelter (2010) give $M_{\text {bol }}$ for V441 Cyg of -5.7, while using Eq. (1) we would determine a value of -4.45 . If this offset were uniform and the scale of Uttenthaler \& Lebzelter (2010) is correct, then R Cyg, T Sgr and S Cas would have bolometric magntidues of approximately -6.0 , though TV Aur would still be one magnitude above this limit.

There are other super-Li rich stars, notably WZ Cas, that present difficulties for HBB theory. Abia et al. (1999) find that WZ Cas (a star on the SC-C border) has $\log \epsilon(\mathrm{Li}) \geq 3.0$ and ${ }^{12} \mathrm{C} /{ }^{13} \mathrm{C}$ of 5 . However, HBB theory predicts that there should be no super-Li rich carbon stars (Boothroyd et al. 1993). Domínquez et al. (2004) find a super-Li rich carbon star in the Draco dwarf galaxy with ${ }^{12} \mathrm{C} /{ }^{13} \mathrm{C}>40$. In fact, GarcíaHernández et al. (2007) argue that there should not be any superLi rich S stars formed by HBB in the Milky Way. A cool bottom burning process (CBP) has been proposed by Wasserburg et al. (1995) to explain abnormal $\mathrm{Li}$ abundances among RGB and low mass AGB stars. However, CBP predicts low values of ${ }^{12} \mathrm{C} /{ }^{13} \mathrm{C}$ as well which is at odds with the findings of García-Hernández et al. (2007) and our findings for S Cas and R Cyg.

Acknowledgements. We thank the outstanding staff at the Pico Veleta Radio Observatory which is operated by the Institut de Radio Astronomy Millimetrique, Grenoble. We also thank A. Ritchey for help in preparing this paper for publication. B. Balick acknowledges the support from grant AST 0808201 from the National Science Foundation. Andrew Vanture's summer research has been supported by the Kenilworth Fund of the New York Community Trust. This work is partially supported by the Spanish MICINN, program CONSOLIDER INGENIO 2010, grant “ASTROMOL” (CSD2009-00038).

\section{APPENDIX: Comparison of predictions from our simple model and from very detailed calculations}

Ramstedt et al. (2009) have recently performed a very sophisticated study of the excitation and emission of CO lines in S-type stars. Level population by collisions with ortho- and para- $\mathrm{H}_{2}$ was taken into account. Two vibrational states, containing a high number of rotational levels, are considered to account for the line excitation due to absorption of FIR photons. The local radiation intensity, also necessary to derive the level populations, 
was accurately (and consistently) calculated in each point of the shell using a Monte Carlo non-local treatment of radiative transfer. These molecular excitation calculations were coupled with a thermodynamical code that estimates the kinetic temperature across the envelope taking into account, in particular, the radiative cooling by molecular emission.

This (particularly complex) code was used to predict intensities of several rotational lines of ${ }^{12} \mathrm{CO}$ and $\mathrm{SiO}$. The comparison of predictions and observations allowed these authors to estimate, among other parameters, the mass-loss rates in a number of S-type stars. The model fitting was optimized minimizing the difference between observations and predictions using a minimum $\chi^{2}$ method.

We have compared the results from our modest code with those obtained by Ramstedt et al. (2009), in the stars which were in common in both studies. For such a purpose, we performed calculations with our code taking the same parameters as these authors, even the ${ }^{12} \mathrm{CO} J=1-0$ intensities when Ramstedt et al. have published data on this line (if not, namely in AA Cam, T Cet, and ST Her, we took our observations). In Table 4, we compare mass-loss estimates $\dot{M}$ obtained from our model in that way (in principle different from those given in Table 2) with the results obtained by Ramstedt et al. (2009) from their sophisticated model fitting. Note that, as discussed in Sect. 3, in some cases it is not clear if the line emission is dominantly optically thin or thick, and the best estimate following our method is an average.

As we can see, our predictions are compatible with those by Ramstedt et al., with differences not larger than $\sim 1 / 3$ of the average values. Also we note that there is no significant trend in our estimates. We have estimated the geometric average (the $n$th square root of the product of $n$ values) of the ratios of both estimates; we find that our estimates are on average $11 \%$ larger than those of Ramstedt et al. The differences are particularly noticeable in the objects in which we could not use ${ }^{12} \mathrm{CO} J=1-0$ data from Ramstedt et al. (in AA Cam, T Cet, and ST Her). If these sources are not taken into account, the systematic difference is just $2 \%$, which suggest that a good deal of the differences we found between both calculations is in fact due to calibration uncertainties.

These differences between calculations from our simple model and from the sophisticated model by Ramstedt et al. (2009) are of the same order of the differences found when estimates of the mass-loss rates from $\mathrm{CO}$ data have been performed by different authors, using different approaches to the problem. We can check the usually obtained differences in the discussion by Ramstedt et al. (2008), who compared calculations and models by different authors to treat $\mathrm{CO}$ line observations. (They also discuss estimates of the mass-loss other than those based on CO lines, which is not relevant for us.) In all cases, the treatments of the $\mathrm{CO}$ excitation and emission were very detailed and complex; original results from a code similar to that in Ramstedt et al. (2009) were also included. Although the assumptions and approaches are very different from model to model, the results were very comparable (except from some surprising cases deeply discussed in that paper). The values of $\dot{M}$ calculated by Ramstedt et al. (2008) were similar to those given by other authors (Groenewegen et al. 1998; Schöier \& Olofsson 2001; Teyssier et al. 2006), but of course not identical, with differences in general between $10 \%$ and $50 \%$.

\section{References}

Abia, C., Boffin, H. M. J., Isern, J., \& Rebolo, R. 1991, A\&A, 245, L1

Abia, C., Pavlenko, Y., \& de Laverny, P. 1999, A\&A, 351, 273

Ake, T. B. 1979, ApJ, 234, 538

Burbidge, E. M., Burbidge, G. R., Fowler, W. A., \& Hoyle, F. 1957, Rev. Mod. Phys., 29, 547

Boothroyd, A. I., Sackmann, I. J., \& Ahrens, S. C. 1993, ApJ, 416, 762

Cameron, A. G. W. 1955, ApJ, 121, 144

Cameron, A. G. W., \& Fowler, W. A. 1971, ApJ, 164, 111

D'Antona, F., \& Mazzitelli, I. 1996, ApJ, 470, 1093

Domíguez, I., Abia, C., Straniero, O., Cristallo, S., \& Pavlenko, Y. 2004, A\&A, 422, 1045

Famaey, B., Jorissen, A., Luri, X., et al. 2005, A\&A, 430, 165

Feast, M. W., Woolley, R., \& Yilmaz, N. 1972, MNRAS, 158, 23

García-Hernández, P. A., García-Lario, P., Plez, B., et al. 2007, A\&A, 462, 711

Groenewegen, M. A. T., van der Veen, W. E. C. J., \& Matthews, H. E. 1998, A\&A, 338, 491

Herwig, F. 2005, ARA\&A, 43, 435

Hinkle, K. H., Hall, D. N. B., \& Ridgway, S. T. 1982, ApJ, 252, 697

Keenan, P. C. 1954, ApJ, 120, 484

Keenan, P. C., \& Boeshaar, P. C. 1980, ApJS, 43, 379

Knapp, G. R., \& Morris, M. 1985, ApJ, 292, 640

Lambert, D. L., Gustafsson, B., Eriksson, K., \& Hinkle, K. H. 1986, ApJS, 62, 373

Lambert, D. L., Smith, V. V., Busso, O., Gallino, R., \& Stranerio, O. 1995, ApJ, 450,320

Loup, C., Forveille, T., Omont, A., \& Paul, J. F. 1993, A\&AS, 99, 291

Luck, R. E., \& Lambert, D. L. 1982, ApJ, 256, 189

Mamon, G. A., Glassgold, A. E., \& Huggins, P. J. 1988, ApJ, 328, 797

Mazzitelli, I., D’ Antona, F., \& Ventura, P. 1999, A\&A, 348, 846

Merrill, P. W. 1922, ApJ, 56, 475

Merrill, P. W. 1952, Science, 115, 484

Nyman, L.-A., Booth, R. S., \& Carlstrom, U. 1992, A\&AS, 93, 121

Olofsson, H., Johansson, L. E. B., Hjalmarson, A., \& Nguyen-Quang-Rieu 1982, A\&A, 107, 128

Palmerini, S., Busso, M., Maiorca, E., \& Guandalini, R. 2009, PASA, 26, 161

Planesas, P., Bachiller, R., Martín-Pintado, J., \& Bujarrabal, V. 1990, ApJ, 351, 263

Plez, B., Smith, V. V., \& Lambert, D. L. 1993, ApJ, 418, 812

Ramstedt, S., Schöier, F. L., Olofsson, H., \& Lundgren, A. A. 2008, A\&A, 487, 645

Ramstedt, S., Schöier, F. L., \& Olofsson, H. 2009, A\&A, 499, 515

Reid, M. J. 1976, ApJ, 207, 784

Sackmann, I. J., \& Boothroyd, A. I. 1992, ApJ, 392, 71

Schöier, F. L., \& Olofsson, H. 2001, A\&A, 368, 969

Smith, V. V., \& Lambert, D. L. 1989, ApJ, 345, L75

Smith, V. V., \& Lambert, D. L. 1990a, ApJ, 361, L69

Smith, V. V., \& Lambert, D. L. 1990b, ApJS, 72, 387

Stephenson, C. B. 1976, Publications of the Warner \& Swasey Observatory, 2, 21

Teyssier, D., Hernandez, R., Bujarrabal, V., Yoshida, H., \& Phillips, T. G. 2006, A\&A, 450, 167

Uttenthaler, S., \& Lebzelter, T. 2010, A\&A, 510, A62

van Leeuwen, F. 2007, A\&A, 474, 653

Vanture, A. D., Smith, V. V., Lutz, J., et al. 2007, PASP, 119, 147

Wallerstein, G. 1985, PASP, 97, 994

Wallerstein, G. 1995, PASP, 97, 1001

Wallerstein, G., \& Morell, O. 1994, A\&A, 281, L37

Wasserburg, G. J., Boothroyd, A. I., \& Sackmann, I. J. 1995, ApJ, 447, L37

Whitelock, P. A., Feast, M. W., \& van Leeuwen, F., 2008, MNRAS, 386, 313

Worley, C. C., Taut, C. A., \& Stancliffe, R. J. 2010, MmSAI, 81, 1016 